\title{
Cent expositions à la Bnu : un début d'historiographie
}

\section{David-Georges Picard}

\section{(2) OpenEdition}

\section{Journals}

Édition électronique

URL : https://journals.openedition.org/rbnu/3424

DOI : 10.4000/rbnu.3424

ISSN : 2679-6104

\section{Éditeur}

Bibliothèque nationale et universitaire de Strasbourg

\section{Édition imprimée}

Date de publication : 1 mai 2011

Pagination : 8-17

ISSN : 2109-2761

\section{Référence électronique}

David-Georges Picard, «Cent expositions à la Bnu : un début d'historiographie », La Revue de la BNU [En ligne], 3 | 2011, mis en ligne le 01 mai 2011, consulté le 08 août 2021. URL : http:// journals.openedition.org/rbnu/3424; DOI : https://doi.org/10.4000/rbnu.3424

\section{(c) (1)(2)}

La Revue de la BNU est mise à disposition selon les termes de la Licence Creative Commons Attribution - Pas d'Utilisation Commerciale - Partage dans les Mêmes Conditions 4.0 International. 


\title{
MANUSCRITS LIVRES ILLUSTRÉS - RELIURES
}

\author{
DU XIII AU XVI SIECLE
}

DE LA BIBLIOTHËQUE DU CARDINAL DE GRANVELLE
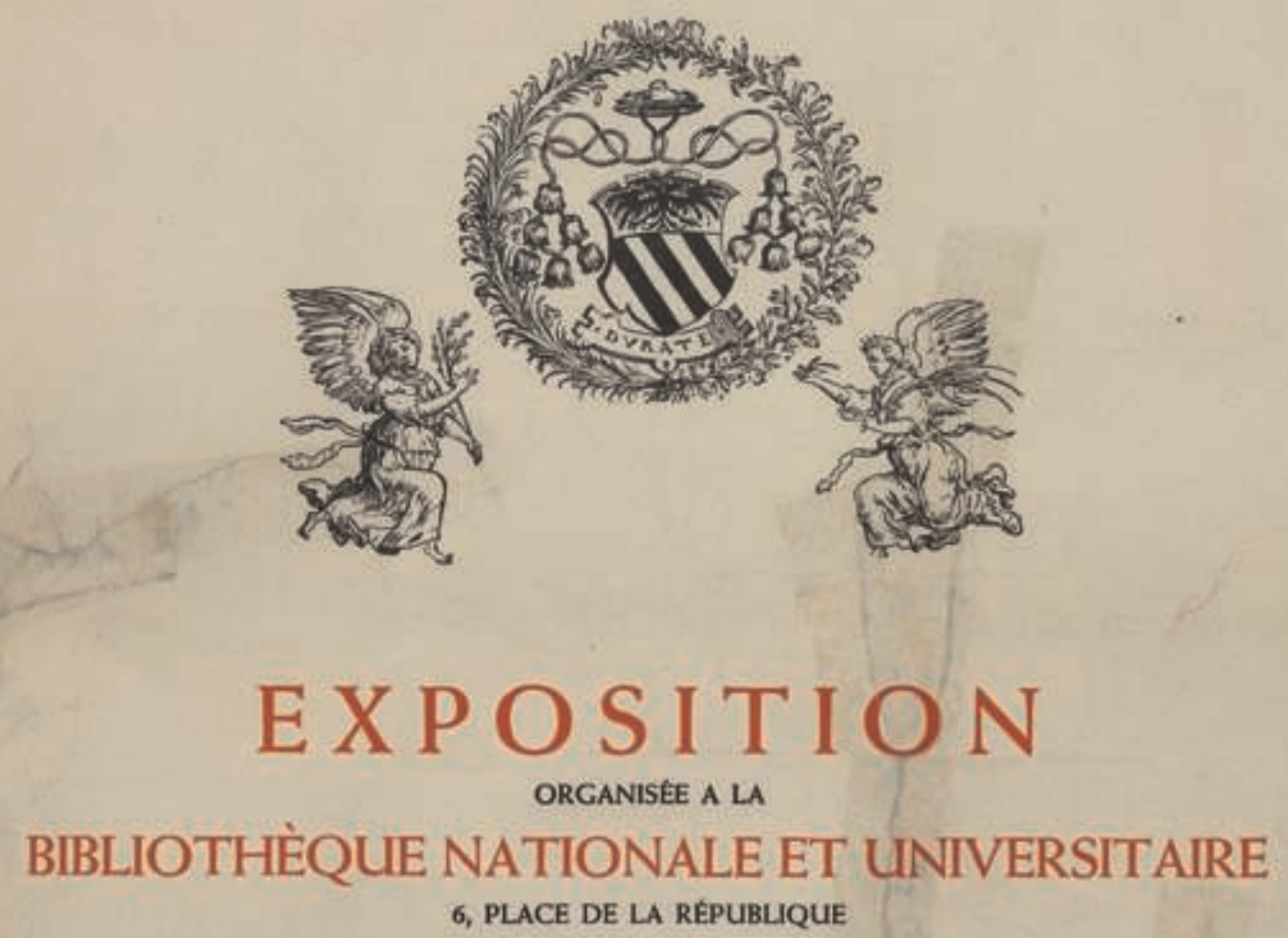

DU 23 NOVEMBRE AU 8 DÉCEMBRE 1951

ENTREE LIBRE TOUS LES JOURS DE 14 A 17 HEURES 


\section{GENT EXPOSITIONS À LA BNU : un début d'historiographie}

A u sortir de la Seconde Guerre mondiale, la Bibliothèque nationale et universitaire de Strasbourg, dont l'enceinte portait encore les stigmates du bombardement du 25 novembre 1944, organisait en ses murs, sous la houlette de Maurice Piquard, son administrateur d'alors, une exposition d'envergure intitulée Manuscrits, livres illustrés, reliures $d u X^{X I I I}{ }^{e}$ au XVI ${ }^{e}$ siècle de la bibliothèque du cardinal de Granvelle (15151586) ${ }^{1}$, célébrant le retour du livre précieux dans l'une des capitales de l'imprimerie. L'inauguration fut soigneusement préparée et Julien Cain, administrateur général de la Bibliothèque nationale et directeur des bibliothèques de France et de la lecture publique, en accepta la présidence. Le discours qu'il prononça donnait ses lettres de noblesse à la démarche de médiation et de valorisation adoptée depuis longtemps par la BNU : « De telles expositions [...] ne nous procurent pas seulement le plaisir le plus délicat, assurait-il, mais bien souvent elles ont permis des confrontations souhaitées par les érudits et ont suscité d'importants travaux. Je souhaite qu'à Strasbourg la tradition s'en établisse. Dans l'ensemble, consacré par le succès, de manifestations de haute culture dont votre ville est le centre et où tous les arts se rejoignent, la bibliothèque doit avoir sa place, et un public toujours plus étendu doit prendre le chemin qui conduit vers ses expositions. C'est là une des fonctions d'une bibliothèque comme la vôtre qui, par sa nature, devrait pouvoir les remplir toutes ${ }^{2}$.
Loin d'être la première de l'établissement, cette exposition consacrée à Granvelle marquait le retour à une politique amorcée pour le moins depuis 1932, sinon depuis l'ouverture en 1895 du bâtiment installé place de la République, anciennement Kaiserplatz, où avait déjà été aménagée une salle d'exposition ${ }^{3}$. La période du Second Empire allemand n'est, pour ce qui concerne l'action culturelle, malheureusement pas documentée dans les archives de l'établissement. Il est vraisemblable néanmoins, au regard des collections de la bibliothèque, que cette salle servit initialement de cabinet de curiosités - elle fut d'ailleurs transformée en petit musée Gobineau quelques années plus tard, comme nous le verrons.

\section{Les lieux d'exposition}

Dans les années 1880-189o, l'exposition comme outil de médiation était loin d'être seulement envisagée dans la très grande majorité des bibliothèques françaises ou allemandes. La BNU a fait exception dès l'érection du bâtiment construit sur mesure pour elle. Un premier espace est réservé à cet effet sur les plans des architectes Skjold Neckelmann et August Hartel. Située au premier étage, cette salle donnait sur la façade nord.

Quelques années après le retour à la France, une nouvelle salle est installée, prise sur le déambulatoire du premier étage donnant à l'ouest, sur la place de la 


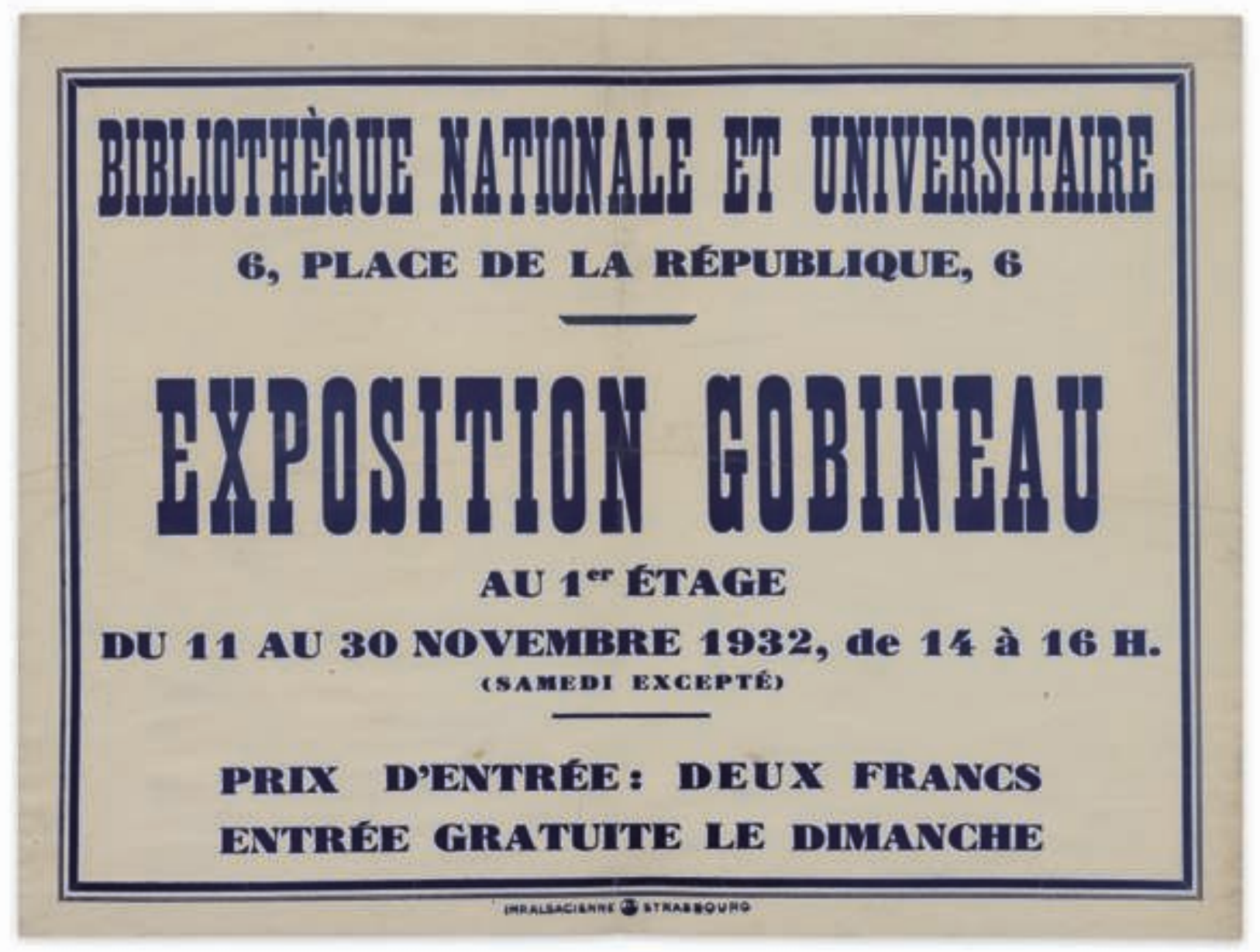


République. Des niches sont creusées dans les murs entre les vastes fenêtres et un plancher est posé. Dans le brouillon non daté d'une lettre adressée au ministère de l'Instruction publique en 1932, l'administrateur Ernest Wickersheimer propose que le vernissage de l'exposition Gobineau soit l'occasion d'inaugurer cette nouvelle salle ${ }^{4}$. Un article d'Étienne Vézian, relatant cet événement, nous renseigne sur l'allure générale de la salle : " La nouvelle salle d'exposition de la Bibliothèque nationale et universitaire a été conçue d'une façon particulièrement heureuse. Les couleurs claires des murs s'harmonisent avec l'éclairage électrique dissimulée (sic) dans le plafond et qui répand une lumière douce sur toute la salle. Les vitrines sont éclairées d'une façon spéciale et les objets exposés ressortent ainsi pour la plus grande joie des yeux. Petit à petit, notre bibliothèque se modernise. Son "outillage" se perfectionne ${ }^{5}$. Les trois expositions suivant celle consacrée à Gobineau auront lieu dans cet espace ${ }^{6}$.

A la suite de la Seconde Guerre mondiale, la BNU, touchée par les bombardements, n'organise plus immédiatement de manifestation d'ampleur. Une exposition " hors les murs " est réalisée en 1949 en partenariat avec les musées du Palais Rohan7, et ce ne sera finalement qu'en 1951 que la BNU, avec l'exposition consacrée à Granvelle, reprendra la main. Celle-ci sera présentée au rez-de-chaussée, à l'angle du bâtiment rue Joffre - place de la République, à l'emplacement de ce qui deviendra, ultérieurement, la salle d'actualité, ainsi qu'en atteste le cliché reproduit p. 13. Salle d'exposition temporaire, puisque les travaux engagés sur les plans de l'architecte François Herrenschmidt permettront de disposer d'une nouvelle salle de $125 \mathrm{~m}^{2}$, située sous la salle de lecture 4, elle-même sous le dôme. L'inauguration de cet espace d'exposition eut lieu le 21 décembre $1954^{8}$, là aussi à l'occasion du vernissage d'une exposition, cette fois-ci d'origine nantaise ${ }^{9}$.

En 2003, avec Impressions d'Europe, les espaces d'exposition migreront une dernière fois pour occuper la salle de lecture 7 au deuxième étage et gagner, ainsi, en surface (avec quelque $440 \mathrm{~m}^{2}$ ), de même qu'en équipement. S'ouvre alors une mutation décisive dans l'action culturelle de l'établissement, avec la possibilité d'organiser des expositions de vastes dimensions, accompagnées de scénographies professionnelles et de catalogues scientifiques richement illustrés. Cette tendance est aujourd'hui perçue comme irréversible et inaugure une nouvelle ère tant dans la conception des expositions à la BNU que dans l'aspect muséal accordé à l'établissement à côté de sa fonction documentaire - aspect muséal qui fait l'objet d'un programme spécifique à l'occasion des travaux de rénovation actuels.

\section{Exposer à la BNU}

Si ce n'est qu'à partir des années 1970 - 1980 que les bibliothèques françaises sont considérées - parfois comme un espace muséal possible, la BNU, sur le modèle de la Bibliothèque nationale à Paris, a développé bien plus tôt une politique culturelle structurée.

A l'instar de la BN, la BNU adopte initialement une démarche dont l'ambition est en priorité la confrontation entre le document et le public. La conception de l'exposition s'appuie essentiellement sur la contextualisation des documents présentés afin de rendre des ensembles thématiques compréhensibles. En ce sens, c'est l'accumulation de pièces exposées qui prévaut entre 1951 et 1980 - il n'est pas rare alors que plus de 300 documents de tous formats soient exposés dans un espace de $125 \mathrm{~m}^{2}$. Accumulation protéiforme, d'ailleurs, où se retrouvent des documents de toute nature (mobilier, sculptures, peintures, armes, tentures, etc.). La contextualisation, le rendu de l' " ambiance " sont l'objectif de cette forme de médiation dont la philosophie générale avait été posée après la Première Guerre mondiale. En introduction du catalogue de l'exposition sur un musée de la littérature, Paul Valéry esquissait ainsi les contours de la notion d'exposition : "Le problème général d'une exposition est de faire voir : il consiste à assembler, à mettre en évidence et en valeur ce qui est ordinairement dispersé, retiré, réservé à quelques-uns, peu accessible, et pour beaucoup, véritablement inconnu ". Il ajoutait quelques lignes plus loin : "Le problème d'exposer n'est qu'une affaire de choix et de mise en ordre et en place ${ }^{10}$. L'explicitation faite par Julien Cain en introduction du même ouvrage nous éclaire tant sur ce que Valéry entendait que sur les motifs de ces choix à la BNU et nous renseigne en partie sur l'état d'esprit des commissaires d'exposition : " La valeur démonstrative du manuscrit est fatalement limitée. Il ne saurait permettre de rendre sensibles les multiples et parfois imperceptibles rapports entre l'œuvre et la réalité. C'est alors que l'image doit s'ajouter au ma- 
nuscrit ${ }^{11}$. La (re-)contextualisation devenait prétexte : on expose alors davantage pour le décorum. Pour l'exposition Granvelle, dont quelques clichés témoignent (voir l'illustration p. 13), sculptures et tableaux notamment un portrait de Nicolas Perrenon par Le Titien emprunté au musée de Besançon - évoquaient l'atmosphère de l'époque du cardinal.

Cette conception de l'exposition a cédé le pas à un souci différent : rendre perceptible au public non pas tant le contexte que le document lui-même. Le discours se fait plus long et le nombre de pièces présentées, en conséquence, plus restreint.

Avec une salle de $440 \mathrm{~m}^{2}$ à partir de

2003, la scénographie devient en

elle-même objet d'exposition

aux côtés des documents

dont le nombre oscille dé-

sormais entre 200 et 300

au maximum.

L'origine des expositions

est souvent à trouver à

l'extérieur de la biblio-

thèque. Dès après 1945,

la BNU est perçue par

son environnement ins-

titutionnel comme un outil

pertinent de médiation, voire

de propagande. Sollicitée très

régulièrement par les institu-

tions européennes, diplomatiques et

politiques, la bibliothèque accueille de

nombreuses expositions, parfois " clé en main ".

La durée courte des expositions - en moyenne trois semaines - permet de juxtaposer les événements à un rythme soutenu. A l'occasion d'expositions internationales à Strasbourg ou de semaines du livre (autrichien, tchèque, roumain...), la BNU expose par exemple des grands noms contemporains de la littérature ou de l'illustration. C'est là un souci constant de l'établissement qui transparaît : l'inscription dans l'actualité artistique et culturelle de Strasbourg. La période des deux mandats de l'administrateur Norbert Schuller, en particulier, fut pour l'établissement l'occasion de se positionner comme interlocuteur tant du ministère français des Affaires étrangères que des ambassades en France et des institutions européennes installées à Strasbourg en exerçant activement une fonction de centre culturel. A partir des années 1980, les sollicitations
" diplomatiques " se firent moins régulières tout en se portant néanmoins sur des projets d'ampleur toujours plus importante (sur Erich Maria Remarque en 2000, l'affiche de la UFA en 2001 ou Stefan Zweig en 2002, pour ne prendre que ces exemples). La dernière exposition en date élaborée dans un cadre international fut Pouchkine illustré ${ }^{12}$, conçue en partenariat avec le Musée d'État Pouchkine de Saint-Pétersbourg et le Musée national Pouchkine de Moscou.

\section{La première exposition française}

A l'instar de celles qui se succéderont jusqu'aux années 1980, la première exposition que la bibliothèque devenue française consacra à la collection du comte Arthur de Gobineau, dont le fonds avait été acquis en $1903^{13}$, fut de courte durée : inaugurée le 10 novembre 1932, elle fut présentée au public jusqu'au 30 novembre. Outre la liste exhaustive des pièces exposées, le catalogue, édité un an après la manifestation ${ }^{14}$, relate sommairement en introduction certains choix scénographiques faits

par Édith Bernardin, conservateur à la BNU et commissaire de l'exposition. Ainsi, nous apprenons par exemple que le parcours s'ouvrait sur le portrait du comte peint par sa maîtresse, la comtesse Mathilde de La Tour en 1876. Suivaient, aux côtés de nombreux manuscrits et imprimés, quelques-unes des pièces les plus "visuelles " du fonds : tableaux, pièces de mobilier, vêtements, photographies, sculptures de la main de Gobineau et d'énigmatiques liasses de correspondances cachetées dont l'ouverture était fixée à 1950. La presse locale et nationale s'est fait d'ailleurs l'écho de ces paquets mystérieux qui paraissent avoir suscité beaucoup d'intérêt ${ }^{15}$. Plus généralement, les éloges concernant la mise en espace des documents sont nombreux. Nous relevons, en particulier, dans un long article du Figaro signé par Jacques Crepet ${ }^{16}$, l'une ou l'autre remarques flatteuses : " Très heureusement aménagée par le distingué administrateur de la 


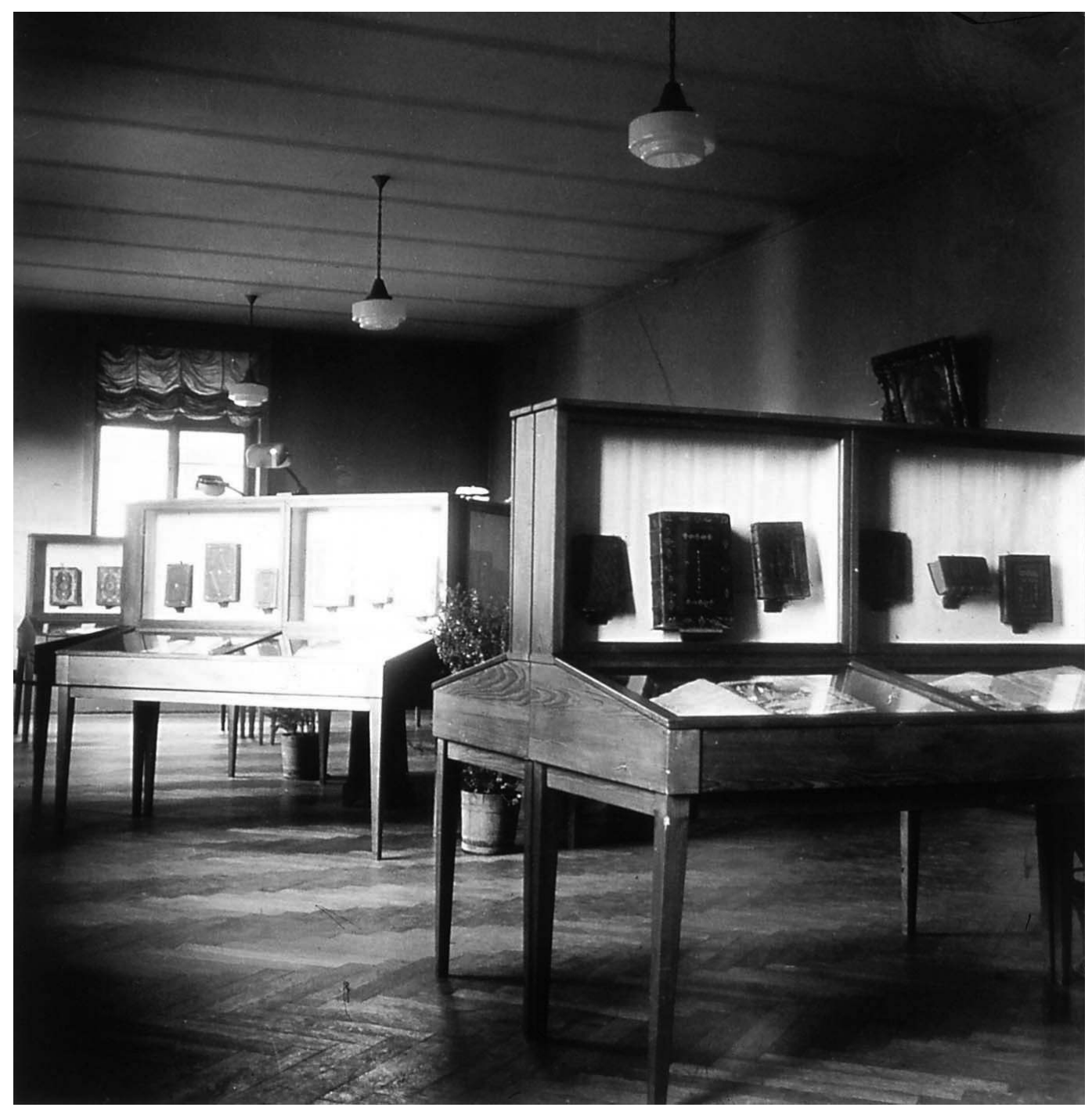
du cardinal de Granvelle. 23 novembre au 8 décembre 1951 (coll. BNU) 
bibliothèque, $\mathrm{M}$. Wickersheimer et par $\mathrm{M}$. Roederer, architecte des bâtiments civils, l'exposition présente un aspect qui séduit l'œil dès l'entrée. Tout autour de la longue salle - on sait que Gobineau, sur le tard, s'était mis à la sculpture - un peuple de statuettes et de bustes qui contribuent à créer une atmosphère d'art et de pensée ".

Cette exposition de la BNU s'insérait alors dans une démarche nationale célébrant le cinquantenaire de la disparition de Gobineau. Un comité de patronage avait été constitué autour d'Édouard Herriot, alors président du Conseil et ministre des Affaires étrangères, et comptant parmi ses membres des personnalités comme Robert de Billy, Joseph Caillaux, Marcel Mauss, Romain Rolland, etc. Ce comité de patronage s'était doté d'un comité de direction chargé de l'exécution de "l'année Gobineau " : la direction en fut confiée au neveu de l'auteur, Clément Serpeille de Gobineau, qui avait rassemblé, là aussi, de grands noms, comme Abel Bonnard, Lucien Descaves, Bernard Faÿ, futur administrateur général de la Bibliothèque nationale durant la Seconde Guerre mondiale, Jean Giraudoux, Jacques de Lacretelle ou Paul Morand ${ }^{17}$.

En tant que propriétaire de la collection Gobineau, la BNU était de facto incontournable pour commémorer ce cinquantième anniversaire. L'établissement fut donc sollicité pour organiser une manifestation autour de ses collections récemment acquises. Et si, lors d'une visite ou de l'inauguration, les personnalités officielles - préfet du Bas-Rhin en tête - et les journalistes s'accordaient à reconnaître la nature peut-être sulfureuse du sujet, mentionnant la situation allemande (Hitler sera chancelier deux mois plus tard), l'unanimité se fit toutefois autour de la complétude et de la présentation du fonds.

Par la grande variété de documents, sa qualité, son exhaustivité, le fonds Gobineau est devenu, dès lors, un des fils d'Ariane de la politique d'action culturelle de la bibliothèque (voir aussi, à ce sujet, le portfolio p. 86). Avant même l'exposition de 1932, il avait déjà fait l'objet, selon les termes négociés par Mathilde de La Tour et à partir de 1906, d'une présentation " dans le cadre d'une pièce particulière aménagée en musée Gobineau " ${ }^{18}$. A de nombreuses reprises, les documents $\mathrm{du}$ fonds figurent dans les expositions ultérieures. La collection, notamment le mobilier, sert tantôt d'outil de mise en scène comme, par exemple, lors de l'exposition Jean Sturm, quand l'humanisme fait école ${ }^{19}$, tantôt est partie intégrante du discours scientifique en tant qu'élément de contextualisation et porteur de contenu.

Ce fut le cas, en particulier, à l'occasion de l'exposition Richard Wagner. Centenaire du Festival de Bayreuth ${ }^{20}$ qui permit, en rappelant les liens qui unissaient les deux hommes, de présenter pour la première fois au public l'air de Klingsor du deuxième acte de Parsifal, page de partition entièrement autographe donnée par Wagner à Gobineau lors de la venue de ce dernier à Wahnfried. Le buste de la Walkyrie sculpté par Gobineau eut aussi droit, aux côtés de 124 portraits exposés (peintures et photographies), à une place de choix.

Ce fut le cas, également, à l'occasion de l'exposition Impressions d'Europe ${ }^{21}$ qui célébrait la richesse des fonds franco-allemands de la bibliothèque et dont le parcours permettait à la collection Gobineau, avec 37 des 307 pièces exposées, de faire bonne figure (un "salon Gobineau " avait été reconstitué pour l'occasion).

\section{Trois succès de l'après-guerre}

En quatre-vingts années de politique culturelle, la BNU aura organisé plus de cent expositions. Les années d'après-guerre notamment correspondent à une période faste, grâce à l'engagement régulièrement salué de trois conservateurs : Édith Bernardin, Lily Greiner (future administratrice) et Madeleine Lang. Malgré leur courte durée, certaines expositions remportent un franc succès. L'exposition Les grandes heures de Strasbourg ${ }^{22}$, par exemple, est organisée à l'occasion del'inauguration du monument au maréchal Leclerc à laquelle se rendait le général de Gaulle, dont la visite à la BNU était espérée. Représentant le président de la République, André Boulloche, ministre de l'Éducation nationale, l'inaugurera. Cette exposition composite retraçant l'histoire de la ville du Ir $^{\text {er }}$ siècle après Jésus-Christ à 1945 au prisme de 347 documents de toute nature (sculptures romaines, pièces archéologiques, manuscrits, imprimés, etc.), provenant des collections de la BNU ou empruntés à de grandes institutions (Bibliothèque Mazarine, Bibliothèque nationale, Bibliothèque universitaire d'Heidelberg, etc.) accueillera plus de 6200 visiteurs. De la même façon, l'exposition Gutenberg et les débuts de l'imprimerie à Strasbourg ${ }^{23}$, élaborée autour de la très riche collection d'incunables de la bibliothèque, 


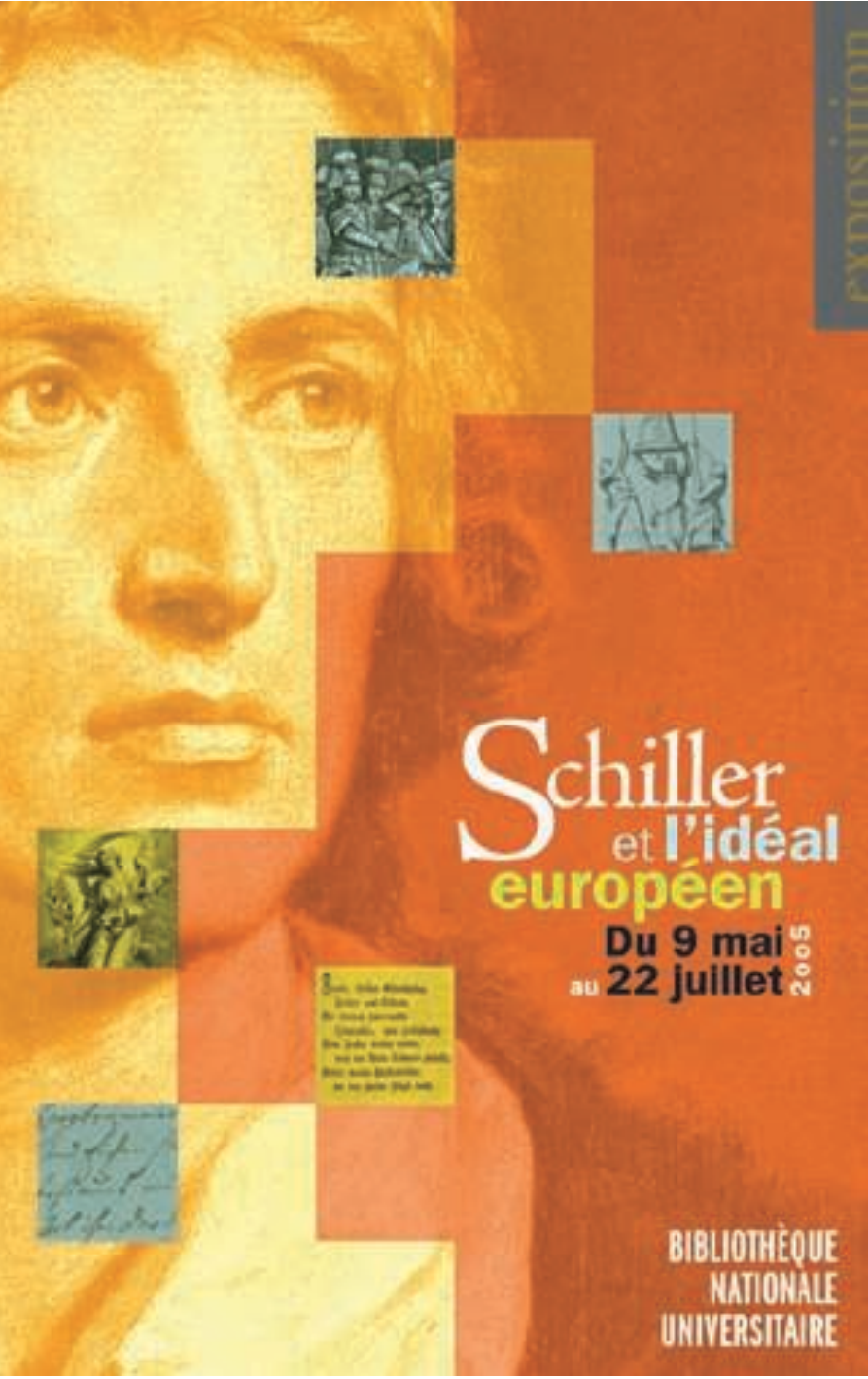

Affiche de l'exposition Schiller et l'idéal européen. 9 mai au 22 juillet 2005 (coll. BNU)

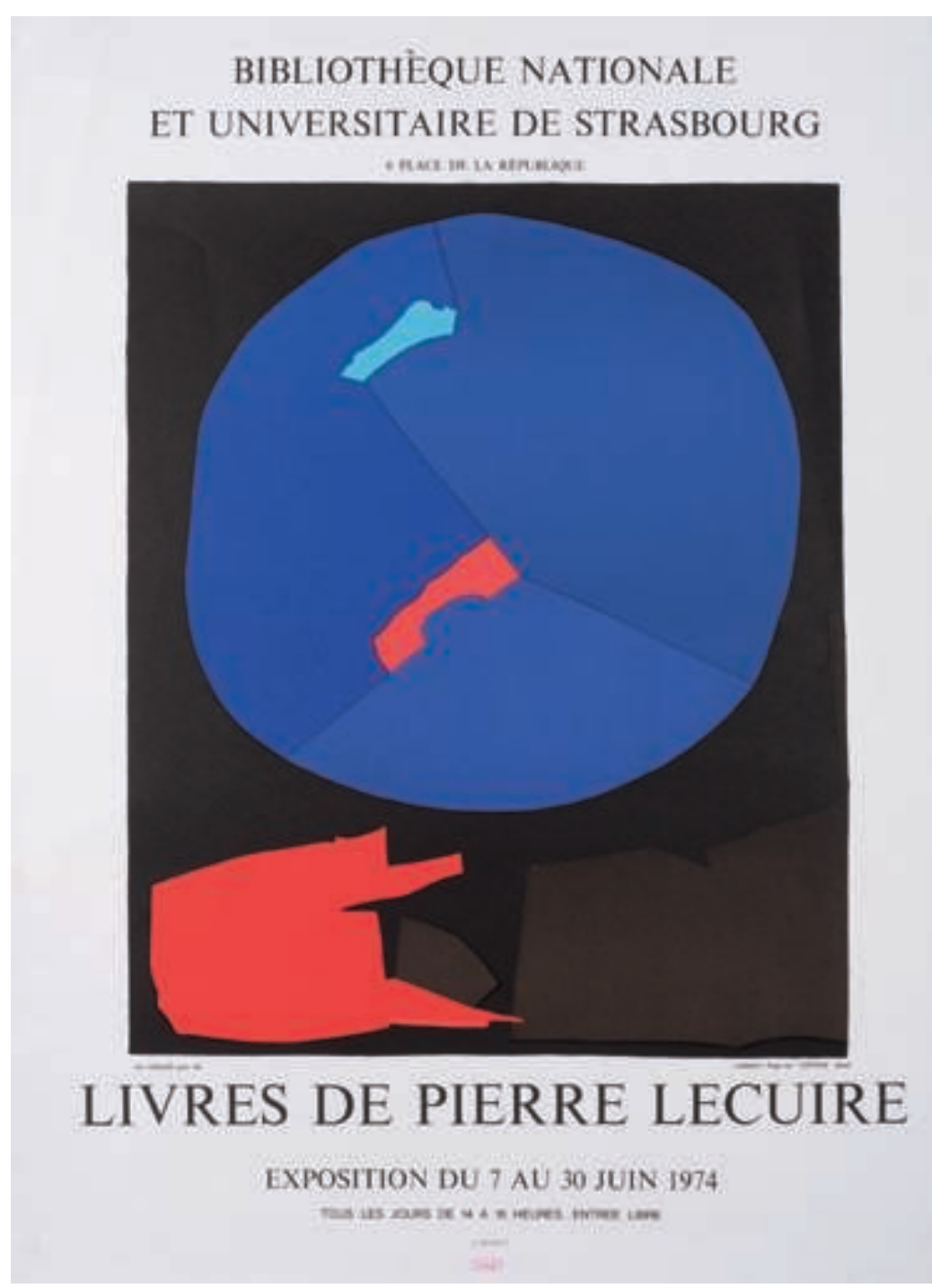

Affiche de l'exposition Livres de Pierre Lecuire. 7 au 30 juin 1974 (coll. BNU) 
recensera 6 ooo entrées. Le goût du public pour la présentation quasi exclusive de ce type de documents est d'ailleurs constant, comme l'a démontré, récemment, l'exposition La route des incunables ${ }^{24}$, soulignant certainement une permanence dans les attentes des usagers.

Peu avant, pour célébrer les vingt ans de la Libération des départements de l'Est de la France, la bibliothèque, en concertation avec un comité national et un comité départemental exécutif (Bas-Rhin, Haut-Rhin et Moselle), élaborait une importante manifestation, pour laquelle un scénographe interviendrait pour la première fois. Résistance et Libération ${ }^{25}$ sera, elle aussi, avec plus de 6 ooo visiteurs, une des brillantes expositions de la bibliothèque qui espérait, une fois encore, la venue du général de Gaulle - espoir une nouvelle fois déçu.

A l'aune des grandes expositions contemporaines d'institutions nationales, expositions courues parfois par des millions de visiteurs, ces chiffres peuvent paraître dérisoires : à cette époque, toutefois, où le public visé est resserré, ces statistiques indiquent que la bibliothèque maintenait et étendait sa situation à la croisée des chemins, entre université et ville, institutions et citoyens.

La politique culturelle de la BNU a été et continue d'être un aspect notable de l'histoire strasbourgeoise et rhénane. Sollicitée par des institutions internationales et des États, sa position singulière dans l'univers des bibliothèques s'en est trouvée consolidée. Nous n'ignorons pas la part qu'a pris la subjectivité dans le choix des manifestations que nous avons souhaité retracer ici. Il n'était bien entendu pas envisageable de les présenter toutes, leur valeur étant d'ailleurs, sans doute, inégale. Quelques jalons sont posés cependant, pour inviter à découvrir plus avant la richesse de ce pan de l'existence de la bibliothèque et tenter plus tard - pourquoi pas ? - la comparaison avec les deux autres établissements français statutairement comparables : la Bibliothèque nationale de France et la Bibliothèque publique d'information.

\section{David-Georges Picard}

\section{Notes}

1 - Manuscrits, livres illustrés, reliures du XIII au XVI siècle de la bibliothèque du cardinal de Granvelle (1515-1586). Du 23 novembre au 8 décembre 1951

2 - Julien Cain, Discours inaugural du 22 novembre 1951, p. 1 et 2. Archives de la BNU, liasse Expositions 1932-1951

3 - Henri Dubled, Histoire de la Bibliothèque nationale et universitaire de Strasbourg. - Strasbourg : Société savante d'Alsace et des régions de l'Est, 1973, p. 18

4 - Archives de la BNU, liasse Expositions 1932-1951

5 - Étienne Vézian, L'inauguration de l'exposition Gobineau à la Bibliothèque nationale et universitaire, in La France de l'Est, 14 novembre 1932

6- Art guarani, du 25 novembre au 10 décembre 1934 ; L'humanisme alsacien - Guillaume Budé, du 25 au 30 avril 1937 ; Jean Sturm, du 15 au 26 novembre 1938

7 - Incunables et livres illustrés strasbourgeois du XV et du XVIe siècles. Palais Rohan, juillet et août 1949

8 - Dernières Nouvelles d'Alsace, $\mathrm{n}^{\circ}$ 6, 7 janvier 1955, p. 19

9 - Autographes européens. Collection La Bouchère. Du 22 décembre 1954 au 31 janvier 1955. Exposition principalement sur panneaux

10 - Paul Valéry, préface, in Julien Cain, Ébauches et premiers éléments d'un musée de la littérature. Exposition internationale des arts et techniques, Paris 1937. - Paris : éditions Denoël, 1938, p. III

11 - Julien Cain, ibidem, introduction, p. XI

12 - Pouchkine illustré, du 12 mai au 20 septembre 2010. Exposition organisée dans le cadre de l'Année France-Russie 2010

13 - Ludwig Schemann, Die Gobineau-Sammlung der Kaiserlichen Universitäts- und Landesbibliothek zu Strassburg, Strasbourg, 1907

14 - Bibliothèque nationale et universitaire de Strasbourg, Exposition à l'occasion du cinquantième anniversaire de la mort d'Arthur de Gobineau (10-30 nov. 1932). Strasbourg, 1933

15 - Voir notamment à ce propos l'article d'Étienne Vézian paru dans La France de l'Est daté du 14 novembre 1932 ainsi qu'un article intitulé L'exposition Gobineau à la bibliothèque nationale et universitaire de Strasbourg, signé Hauth et paru dans Le Temps du 10 décembre 1932

16 - Jacques Crepet, L'exposition Gobineau à Strasbourg, in Le Figaro, Paris, 19 novembre 1932, p. 5

17 - Dans les archives de la BNU, la liste des membres de l'un et l'autre comité a été conservée dans la liasse Expositions 1932-1951.

18 - Isabelle Didier, Le fonds Gobineau, in Impressions d'Europe. Catalogue de l'exposition. - Strasbourg : Bibliothèque nationale et universitaire, 2003, p. 65

19 - Jean Sturm, quand l'humanisme fait école. Du 11 octobre au $1^{\text {er }}$ décembre 2007

20 - Richard Wagner. Centenaire du Festival de Bayreuth. Du 8 juin au 29 juin 1976

21 - Impressions d'Europe. Du 6 novembre 2003 au 24 janvier 2004

22 - Les grandes heures de Strasbourg. Du 23 novembre au 13 décembre 1959

23 - Gutenberg et les débuts de l'imprimerie à Strasbourg. Du 2 mars au 22 mars 1968

24 - Premières impressions à travers l'Europe, de Venise à Louvain. La route des incunables. Exposition présentée du 17 juillet au 16 septembre 2007

25 - Résistance et Libération. Du 22 novembre au 20 décembre 1964 


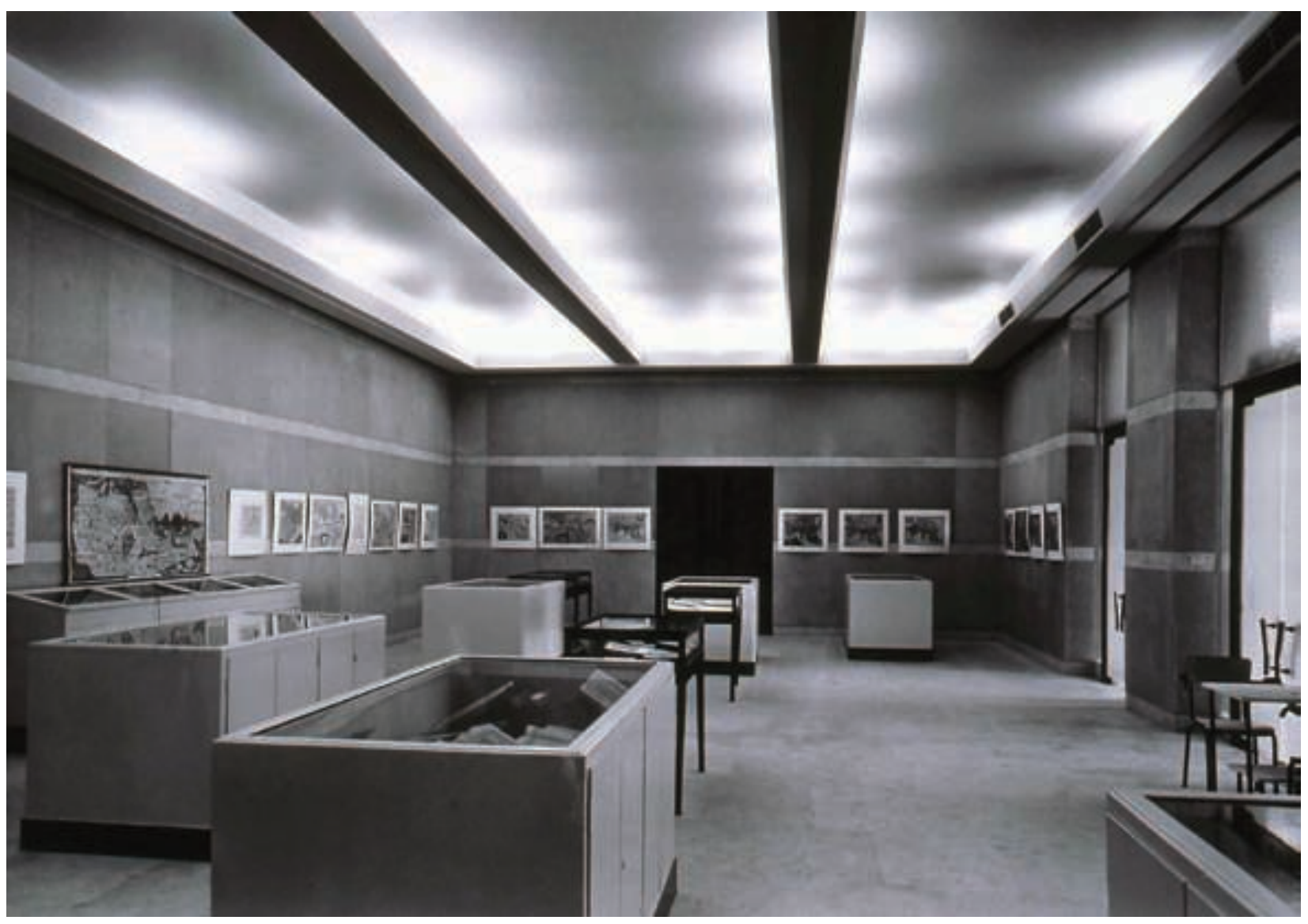

